\title{
Nuclear recoil measurement in CsI(Tl) crystal for Cold Dark Matter detection
}

\author{
M.Z. Wang ${ }^{\text {a }}$, Q. Yue ${ }^{\text {b }}$, J.R. Deng ${ }^{\text {c }}$, W.P. Lai ${ }^{\text {d,e }}$, H.B. Li ${ }^{\text {a,d }}$, J. Li $^{\text {b }}$, Y. Liu ${ }^{\text {b }}$, B.J. Qi ${ }^{\text {c }}$, \\ X.C. Ruan ${ }^{\mathrm{c}}$, C.H. Tang ${ }^{\mathrm{a}}$, H.Q. Tang ${ }^{\mathrm{c}}$, H.T. Wong ${ }^{\mathrm{d}, *}$, S.C. Wu ${ }^{\mathrm{a}}$, B. Xin ${ }^{\mathrm{c}}$, Z.Y. Zhou ${ }^{\mathrm{c}}$ \\ a Department of Physics, National Taiwan University, Taipei, Taiwan \\ ${ }^{\mathrm{b}}$ Institute of High Energy Physics, Beijing, China \\ ${ }^{\mathrm{c}}$ Department of Nuclear Physics, Institute of Atomic Energy, Beijing, China \\ $\mathrm{d}$ Institute of Physics, Academia Sinica, Taipei, Taiwan \\ e Department of Management Information Systems, Chung Kuo Institute of Technology, Taipei, Taiwan
}

Received 16 January 2002; received in revised form 19 April 2002; accepted 22 April 2002

Editor: J.P. Schiffer

\begin{abstract}
The potential merits of $\mathrm{CsI}(\mathrm{Tl})$ scintillating crystals for Dark Matter experiments make it a subject of recent interest. The scattering signatures by neutrons on a $\mathrm{CsI}(\mathrm{Tl})$ detector were studied using a neutron beam generated by a 13 MV Tandem accelerator. The energy spectra of nuclear recoils from $7 \mathrm{keV}$ to $132 \mathrm{keV}$ were measured, and their quenching factors for scintillating light yield were derived. The data represents the first confirmation of the Optical Model predictions on neutron elastic scatterings with a direct measurement on the nuclear recoils of heavy nuclei. ๑ 2002 Elsevier Science B.V. All rights reserved.
\end{abstract}

PACS: 25.40.Dn; 95.35.+d; 29.40.Mc

Keywords: Neutron elastic scattering; Dark matter; Scintillation detectors

\section{Introduction}

The detection of Dark Matter and the studies of their properties [1] are of fundamental importance in particle physics and cosmology. The Weakly Interacting Massive Particles (WIMPs) are good candidates for "Cold" Dark Matter, and their experimental searches have gathered a lot of interests in recent

\footnotetext{
* Corresponding author.

E-mail address: htwong@phys.sinica.edu.tw (H.T. Wong).
}

years. The most promising avenue is to detect the nuclear recoil signatures due to elastic scatterings of WIMPs on the target isotopes. The typical energy depositions are only of the order of $10 \mathrm{keV}$, imposing big experimental challenges in terms of the detection of weak signals as well as background control at low energy close to detection threshold. A wide spectrum of experimental techniques is being pursued [2].

The DAMA experiment observed an annual modulation of nuclear recoil events [3] with $\mathrm{Nal}(\mathrm{Tl})$ scintillating crystal detectors, which can be interpreted as positive evidence of WIMPs due to the difference of 
the relative velocities of the Earth from the Halo sea within the year. However, results from the CDMS experiment [4] based on cryogenic technique were interpreted as in contradiction to the DAMA results when a particular model framework was considered.

There are potential merits of using $\mathrm{CsI}(\mathrm{Tl})$ scintillating crystals [5] for WIMP search and other lowenergy low-background experiments [6,7]. An experiment towards $200 \mathrm{~kg}$ of $\mathrm{CsI}(\mathrm{Tl})$ crystal scintillators to study low energy neutrino interactions at the KuoSheng power reactor is being pursued [7], while the adaptation of the crystal for Dark Matter searches are the focus of several on-going projects $[8,9]$ and an approved experiment [10].

One of the key issues to realize a Dark Matter search experiment with $\mathrm{CsI}(\mathrm{Tl})$ crystal scintillator is the studies of the experimental signatures of nuclear recoils due to WIMP-nuclei elastic scatterings. Nuclear recoils produce large charge density $(\mathrm{d} E / \mathrm{d} x)$. The scintillating light output is expected to be reduced ("quenched") while the timing profile of the pulse is different relative to the same energy deposition by minimum ionizing particles [11].

These experimental observables are the same as those due to nuclear recoil events produced by elastic scattering of neutrons on nuclei. The quenching factors measurement is reported in this Letter. They extend and improve on the work from the other recent measurements on $\mathrm{CsI}(\mathrm{Tl})$ [8,9], as well as on other scintillating crystals such as $\mathrm{NaI}(\mathrm{Tl})$ and $\mathrm{CaF}_{2}(\mathrm{Eu})$ [12].

\section{Experimental setup and procedures}

The experiment was performed at HI-13 Tandem accelerator at the China Institute of Atomic Energy (CIAE) in Beijing. A pulsed deuteron beam at $5.6 \mathrm{MeV}$ interacted with a deuterium gas target in a cell $(1 \mathrm{~cm}$ in diameter and $3 \mathrm{~cm}$ in length) at a pressure of 6 bar. Neutrons at $8.0 \mathrm{MeV}$ kinetic energy with an RMS spread of $0.2 \mathrm{MeV}$ were produced at zero degree. The CsI(Tl) sample was located 2.02 meters away from the deuterium target. Neutrons at zero degree were selected by a $32 \mathrm{~mm}$ by $35 \mathrm{~mm}$ collimator of length $1.2 \mathrm{~m}$. The collimator were surrounded by shielding materials like iron, paraffin, lead and polyethylene to reduce background. During this data tak- ing, the repetition rate of the pulsed beam was set at $4 \mathrm{MHz}$ with $1 \mathrm{~ns}$ width and the average beam current was at $1 \mu \mathrm{A}$.

The scattering target, which also functioned as a detector, was a $\operatorname{CsI}(\mathrm{Tl})$ crystal scintillator ${ }^{1}$ with diameter $3 \mathrm{~cm}$ and length $3 \mathrm{~cm}$ wrapped with Teflon sheets, aluminum foil and black plastic tape. To minimize supporting structures, the detector was hung at the correct position by a piece of string. The readout was achieved by a $29 \mathrm{~mm}$ diameter photo-multiplier tube (PMT). ${ }^{2}$ The PMT signals passed through an amplifier and shaper and were digitized by a $20 \mathrm{MHz}$, 8-bit, Flash Analog-to-Digital Converter (FADC) [13]. The electron-equivalence response of the $\mathrm{CsI}(\mathrm{Tl})+$ PMT detector was studied with standard ${ }^{109} \mathrm{Cd}$ and ${ }^{133} \mathrm{Ba} \gamma$-sources. In addition, measurements of the PMT signals with an LED pulsed at single photoelectron intensity provided the calibration of 4 photoelectrons per $\mathrm{keV}$ of electron-equivalence energy.

Neutron tag was provided by liquid scintillator ${ }^{3}$ detectors $105 \mathrm{~mm}$ in diameter and $50 \mathrm{~mm}$ in length equipped with $\mathrm{PMT}^{4}$ readout and placed at various distance $(r)$ and scattering angle $(\theta)$ from the target. The liquid scintillator provides pulse shape discrimination (PSD) capabilities for $n-\gamma$ separation by commercial electronics. ${ }^{5}$ The neutron tag signal was used to define the "START" timing for the time of flight (TOF) system. The pulsed deuteron "pick-off" signal provided by the accelerator was delayed and used as "STOP" for the TOF. The timing was arranged such that the STOP signal would always arrive before the next neutron pulse, so as to eliminate confused and accidental triggers. The $(r, \theta)$ configurations were chosen to optimize the TOF resolution to differentiate neutron elastic scatterings off protons from those of heavier nuclei in the wrapping materials and the CsI target.

The FADC recorded data continuously in a circular buffer of $4 \mathrm{k}$ size. A START-STOP sequence from the TOF system initiated a trigger which stopped the FADC digitization after $25 \mu \mathrm{s}$. The pulse shape

\footnotetext{
1 Producer: Unique Crystal, Beijing.

2 CR110, Hamamatsu Photonics, China.

3 Co-261, ST-451.

4 Philips XP-2041.

5 CANBERRA 2160A.
} 


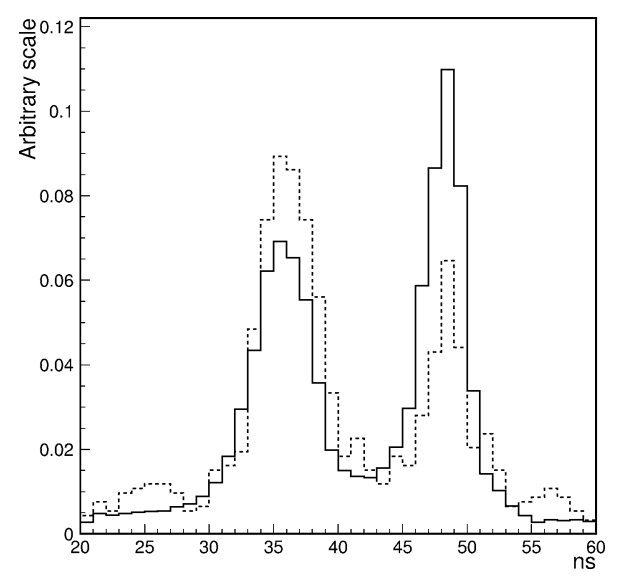

Fig. 1. The TOF spectra at $50^{\circ}$ scattering angle, for configurations with wrapping material only (dashed histogram) and with $\mathrm{CsI}(\mathrm{Tl})$ target (solid histogram).

from the $\mathrm{CsI}(\mathrm{Tl})$ crystal was recorded for a pretrigger and post-trigger periods of $5 \mu$ s and $25 \mu$ s, respectively. The typical data taking rate with such trigger conditions was $4 \mathrm{~Hz}$.

Data taking at each scattering angle was complemented by a measurement of the TOF background spectrum with empty target which was made of the CsI(Tl) wrapping materials only. Displayed in Fig. 1 is the TOF spectra at $50^{\circ}$ with empty and complete target. Faster neutrons have earlier START pulses resulting in larger TOF values. The "slower" $\left(P_{\mathrm{p}}\right.$ at 36 ns) and "faster" ( $P_{\mathrm{N}}$ at $\left.48 \mathrm{~ns}\right)$ peaks are due to neutron elastic scattering off protons and the heavier nuclei such as $\mathrm{C}, \mathrm{Al}, \mathrm{F}, \mathrm{Cs}$ and $\mathrm{I}$, respectively. The relative intensities between the two peaks and among the two target configurations are consistent with the expectations derived from the ratios of target compositions and their respective neutron scattering cross sections. The flat background is due to random coincidence. It can be seen that with the CsI(Tl) target in place, $P_{\mathrm{N}}$ for neutron scattering off heavier nuclei becomes enhanced. The normalized difference between the two TOF spectra are the recoil events from Cs and I.

The reconstructed energy of the $\mathrm{CsI}(\mathrm{Tl})$ target is defined as the pedestal-subtracted integrated area of the FADC pulse, where the pedestal measurements are derived from the time period before the accuratelyknown "time-zero" $\left(t_{0}\right)$ of the $\mathrm{CsI}(\mathrm{Tl})$ events during the same data taking. This pedestal-subtraction would effectively eliminate the bias from pile-up effects due

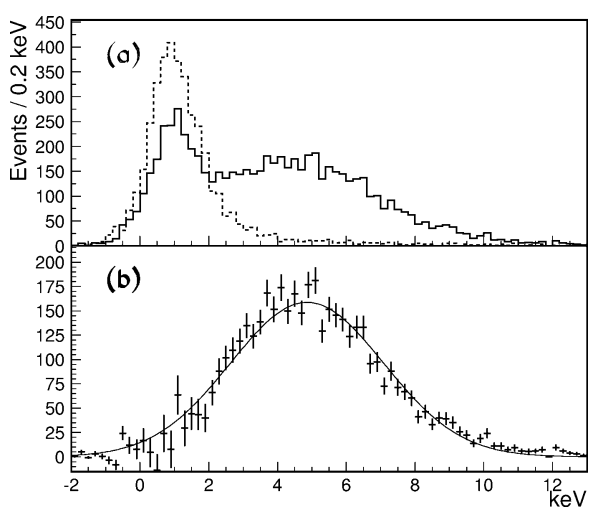

Fig. 2. The $\mathrm{CsI}(\mathrm{Tl})$ recoil energy spectra at $50^{\circ}$ scattering angle for (a) events at $P_{\mathrm{N}}$ (solid histogram) and $P_{\mathrm{p}}$ (dashed histogram), and (b) the subtracted excess fitted to a Gaussian.

to accidental low energy $\gamma$ 's along the beam line. A restricted integration time window of $2.5 \mu$ s starting from $t_{0}$ is adopted for further optimizing the energy resolution.

Events are categorized into two groups by the central value between the two TOF peaks and their corresponding nuclear recoil energy spectra are compared. Using the data set at $50^{\circ}$ as illustrations, the energy spectra are displayed in Fig. 2a for events under the $P_{\mathrm{N}}$ (solid histogram) and $P_{\mathrm{p}}$ (dashed histogram) peaks where the null events consistent with the pedestal background are rejected. The $P_{\mathrm{p}}$ events are triggered by proton recoils from the wrapping materials while having finite signals at the $\mathrm{CsI}(\mathrm{Tl})$ target due to accidental $\gamma$ 's. The spectrum shows a distinct peak with long tail on the high-energy side.

This spectrum, scaled by a normalization factor $f$, should also contribute to the background in the $P_{\mathrm{N}}$ spectrum which originated from neutrons scattering off the heavier nuclei in the wrapping materials. The normalization factor $f$ is the ratio of the $P_{\mathrm{p}}$ to $P_{\mathrm{N}}$ peaks in the empty target configuration in Fig. 1. The detector response for $\mathrm{CsI}(\mathrm{Tl})$ nuclear recoil events can then be derived by subtracting $f$ times the dashed histogram from the solid one in Fig. 2a. Displayed in Fig. $2 b$ is the resulting spectrum with a Gaussian fit.

\section{Measurement results}

The CsI recoil spectra obtained by the analysis scheme discussed in Section 2 were evaluated from the 
Table 1

Measured results on electron-equivalence light yield (E.E.) and quenching factor Q.F. of the neutron elastic scatterings from the CsI(Tl) target, for different neutron scattering angle $(\theta)$ targetdetector distance $(r)$ and nuclear recoil energy $(T)$. Errors shown are combined systematic and statistical uncertainties

\begin{tabular}{lrclr}
\hline$\theta\left(^{\circ}\right)$ & $r(\mathrm{~cm})$ & $T(\mathrm{keV})$ & E.E. $(\mathrm{keV})$ & Q.F. $(\%)$ \\
\hline 20 & 228 & 7.3 & $1.25 \pm 0.20$ & $17.1 \pm 2.7$ \\
30 & 133 & 16.2 & $2.26 \pm 0.27$ & $14.0 \pm 1.7$ \\
40 & 100 & 28.4 & $3.57 \pm 0.57$ & $12.6 \pm 2.0$ \\
50 & 89 & 43.3 & $4.86 \pm 0.49$ & $11.2 \pm 1.1$ \\
60 & 60 & 60.6 & $6.53 \pm 0.65$ & $10.8 \pm 1.1$ \\
65 & 63 & 70.0 & $7.18 \pm 0.72$ & $10.3 \pm 1.0$ \\
80 & 70 & 100 & $10.2 \pm 1.0$ & $10.2 \pm 1.0$ \\
95 & 68 & 132 & $13.4 \pm 1.3$ & $10.1 \pm 1.0$ \\
\hline
\end{tabular}

measurements of eight different scattering angles. The background levels away from the signal peaks after subtraction are always consistent with zero, showing the validity of this procedure. The mean light output and the number of observed events were derived from the centroid and area of Gaussian fits to the recoil spectra, respectively. The results are summarized in Table 1.

The nuclear recoil energy $(T)$ is related to the neutron scattering angle $(\theta)$ by a simple kinematical formula:

$T=\frac{2 A}{(1+A)^{2}}(1-\cos \theta) E_{\mathrm{n}}$,

where $A$ is the target atomic mass and $E_{\mathrm{n}}$ is the incident neutron energy. The neutron scattering angle can be considered to be the same in this case in both the laboratory and center-of-mass systems. Comparisons of the nuclear recoil spectra with those due to calibration sources allow the evaluation of the quenching factors, defined as the mean light yield from nuclear recoils versus that from $\gamma$ sources.

As mentioned, a restricted integration time of $2.5 \mu$ s was adopted for the energy definition of the $\mathrm{CsI}(\mathrm{Tl})$ events, as compared to a typical complete light collection time of $12.5 \mu \mathrm{s}$. A correction factor of 1.15 is applied to the nuclear recoils light yield to account for this partial summation. This factor was derived by averaging and comparing a large number of events due to nuclear recoils and ${ }^{109} \mathrm{Cd} \gamma$-source. The estimated uncertainty is $10 \%$, based on studies with different integration time window from $1.5 \mu \mathrm{s}$ to $12.5 \mu \mathrm{s}$. The pile-up effects are accounted for by pedestal-subtraction, and the residual errors are less than $0.02 \mathrm{keV}$ for all data points. The TOF triggers are independent of the $\mathrm{CsI}(\mathrm{Tl})$ light output, and therefore ensure that all events are detected. The data point at $20^{\circ}$ corresponds to a light yield of $1.25 \mathrm{keV}$ electron-equivalence or 5 photo-electrons, such that a detection efficiency of better than $99 \%$ is achieved.

The uncertainty of the mean nuclear recoil energy due to finite detector size is at most $2 \%$ for different scattering angles. This is checked by calculating this mean energy with proper weights of angular distributions, detector acceptance and the spread in neutron energy. A shift of detector at $1 \mathrm{~mm}$ scale has negligible effect on the central value of nuclear recoil energy. This uncertainty is small compared to that of the electron-equivalence light yield measurements shown in Table 1. The beam current was stable at the $1 \%$ level, as indicated by the stable data acquisition rates and measurements from beam monitor counters. The statistical and systematic uncertainties are then combined in quadrature to give the total uncertainties for each data point. The results were cross-checked with an alternative pulse shape analysis algorithms [14]. Instead of statistical subtraction, nuclear recoil events were selected based on their different pulse shapes compared to $\gamma$-background. The systematics of both procedures are different and the consistency levels among them provide an estimate of additional systematic uncertainties which amounts to $16 \%$ at the lowest energy point at $20^{\circ}$.

The measured quenching factors are depicted in Fig. 3a, while the variation of electron-equivalence light yield (the "visible" energy) versus recoil energies are shown in Fig. 3b. Previous measurements [8,9] are overlaid for comparison. There is a clear trend of less quenching towards low energy points. This measurement achieved a lower threshold and with improved uncertainties. The quenching factors are of the similar range to those for $\mathrm{NaI}(\mathrm{Tl})$, which are typically $25 \%$ for $\mathrm{Na}$ and $8 \%$ for I from Ref. [15]. A linear fit with a finite intercept is in excellent agreement with our data set between the electron-equivalence light yield and the nuclear recoil energy. Data from Ref. [8] shows more quenching and deviates from the linear regime at high energy. This linearity in the CsI(Tl) response is different from the highly non-linear characteristics due to threshold effects observed in liq- 


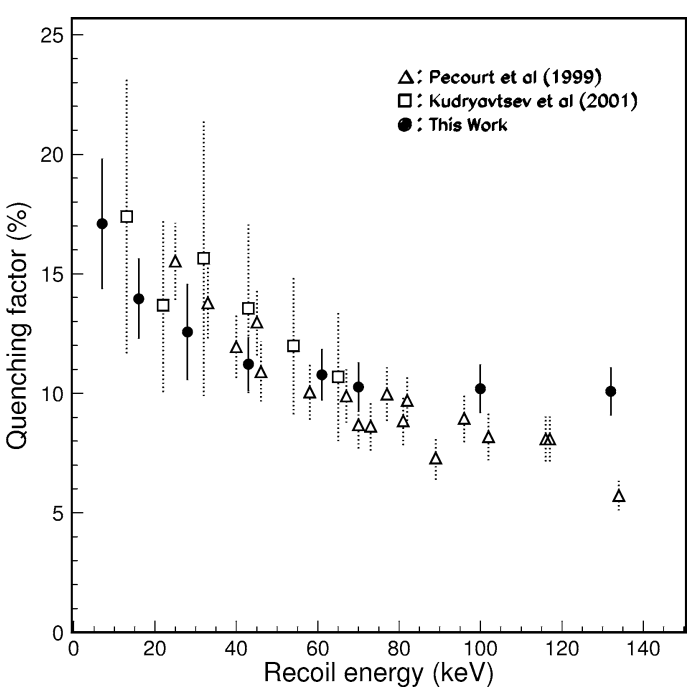

(a)

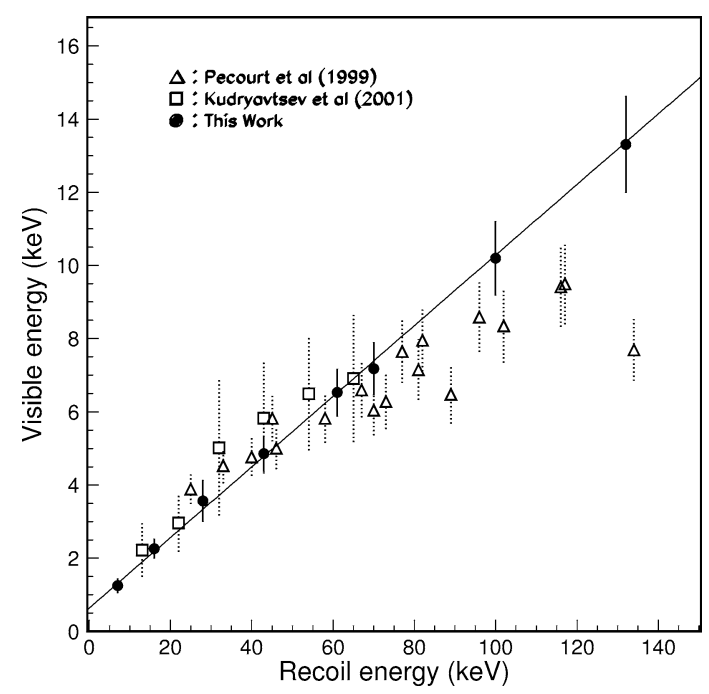

(b)

Fig. 3. (a) The quenching factors and (b) electron-equivalence light yield versus recoil energy measured in this work (black circles), as well as in Ref. [8] (open triangles) and Ref. [9] (open squares).

uid scintillator [16] at the same range of recoil energy.

To derive the differential cross sections from the observed number of events, the data taking time, the acceptance (proportional to $r^{-2}$ ) and the efficiency correction factors have to be evaluated. The efficiency factors are due to the TOF selection criteria and background subtraction scheme. It is close to $100 \%$ for the well-separated TOF peaks, but is only $42 \%$ at $20^{\circ}$ where there are overlap between the TOF peaks. The contributions from inelastic scattering leading to the low excited levels for ${ }^{133} \mathrm{Cs}$ and ${ }^{127} \mathrm{I}$ are expected to be less than $0.1 \%$ relative to the elastic processes.

After all the correction factors are taken into account, the measured angular distribution for neutron elastic scatterings off $\mathrm{Cs}$ and I is displayed in Fig. 4. The uncertainties are dominated by statistical errors from the subtraction procedures. The shape of the solid curve is due to the evaluated neutron elastic scattering cross sections on ${ }^{133} \mathrm{Cs}$ and ${ }^{127} \mathrm{I}$ from the ENDF/B-VI library [17] which originates from the Optical Model calculation. There is excellent agreement between the recoil angular distribution from this measurement and the predictions from the Optical Model. In particular, this is a kinematical regime where the size of the nuclei is comparable

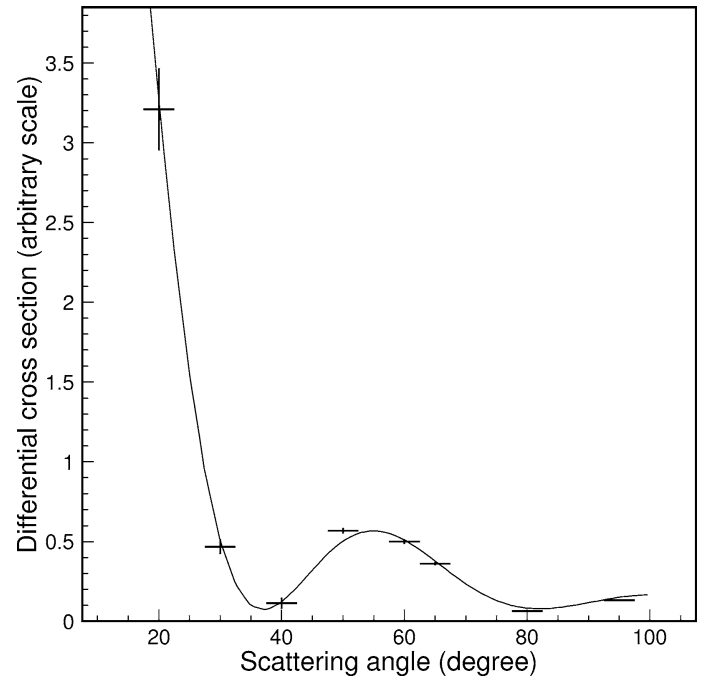

Fig. 4. The measured nuclear recoil differential cross sections in $\mathrm{CsI}(\mathrm{Tl})$, superimposed with the Optical Model prediction.

to the wavelength of the incident neutrons, and the diffraction pattern is well-reproduced by the data. The differential cross-section measurements demonstrate the validity of the analysis procedures at all angles, and provides an additional consistency check on the light yield results in Fig. 3. 


\section{Discussion and summary}

In this Letter, we present a new measurement on the quenching factors of $\mathrm{Cs}$ and $\mathrm{I}$ in a CsI(Tl) crystal scintillator. Lower threshold and improved accuracies are achieved compared to previous measurements. The measured differential cross-section of neutron scattering from I and Cs is in excellent agreement with Optical Model derivations, and represents the first confirmation of the Optical Model on neutron elastic scattering cross-sections with a direct measurement of nuclear recoils from heavy nuclei. That is, the neutrons do elastically scatter off from the nuclei but not via some anomalous processes. The recoil differential cross sections and the quenching factors are relevant to the studies of radiation damage in materials.

By optimizing the detector geometry and using green-extended photo-cathode, a detector with several $\mathrm{kg}$ modular mass and a light yield of a few photoelectron per keV can be realized [10]. The background considerations for $\mathrm{CsI}(\mathrm{Tl})$ detectors in terms of ambient radioactivity and intrinsic radio-purity are discussed in Refs. $[6,7,18]$. Levels of better than the $10^{-12} \mathrm{~g} / \mathrm{g}$ level in concentration for the ${ }^{238} \mathrm{U}$ and ${ }^{232} \mathrm{Th}$ series have been demonstrated, assuming secular equilibrium. The potential problem of the internal ${ }^{137} \mathrm{Cs}$ contaminations can be overcome via selection of clean ore materials and careful chemical processing and purification treatment [10]. Given that the PSD capabilities for $\mathrm{CsI}(\mathrm{Tl})$ are better than those of $\mathrm{NaI}(\mathrm{Tl})$ and that there is matured experience of scaling up the $\mathrm{CsI}(\mathrm{Tl})$ detector to multi-ton systems, there are potentials of further improvements in the current sensitivities, and in the case of positive results, performing an accurate measurement of the annual modulation.

\section{Acknowledgements}

The authors would like to thank Drs. K.W. Ng, S.K. Kim and Y.D. Kim for fruitful discussions and helpful comments, and are grateful to the technical staff from CIAE and IHEP for operating the accelerator. This work was supported by contracts $\mathrm{CosPa}$ 89-N-FA01-1-4-2 from the Ministry of Education, Tai- wan, NSC 89-2112-M-001-056 and NSC 90-2112-M001-037 from the National Science Council, Taiwan, and NSF19975050 from the National Science Foundation, China. The support of H.B. Li is from contracts NSC 90-2112-M002-028 and MOE 89-N-FA011-0 under Prof. P.W.Y. Hwang.

\section{References}

[1] For an overview, see, for example, E. Kolb, M. Turner, The Early Universe, Addison-Wesley, 1989, and references therein.

[2] For a recent review, see, for example, A. Morales, Nucl. Phys. B (Proc. Suppl.) 87 (2000) 477, and references therein.

[3] R. Bernabei et al., Phys. Lett. B 509 (2001) 197, and references therein.

[4] R. Abusaidi et al., Phys. Rev. Lett. 84 (2000) 5699, and references therein.

[5] H. Grassmann, E. Lorentz, H.G. Moser, Nucl. Instrum. Methods 228 (1985) 323;

P. Schotanus, R. Kamermans, P. Dorenbos, IEEE Trans. Nucl. Sci. 37 (1990) 177.

[6] H.T. Wong et al., Astropart. Phys. 14 (2000) 141.

[7] H.T. Wong, J. Li, Mod. Phys. Lett. A 15 (2000) 2011; H.B. Li et al., TEXONO Collaboration, Nucl. Instrum. Methods A 459 (2001) 93;

Y. Liu et al., TEXONO Collaboration, Nucl. Instrum. Methods A 482 (2002) 125.

[8] S. Pecourt et al., Astropart. Phys. 11 (1999) 457.

[9] V.A. Kudryavtsev et al., Nucl. Instrum. Methods A 456 (2001) 272.

[10] H.J. Ahn et al., Technical Design Report, KIMS Collaboration, 2001;

H. Park et al., nucl-ex/0202014, submitted to Nucl. Instrum. Methods A.

[11] See, for example, J.B. Birks, Theory and Practice of Scintillation Counting, Pergamon, 1964.

[12] D.R. Tovey et al., Phys. Lett. B 433 (1998) 150.

[13] W.P. Lai et al., TEXONO Collaboration, Nucl. Instrum. Methods A 465 (2001) 550.

[14] Q. Yue et al., Nucl. Instrum. Methods A, in preparation.

[15] G. Gerbier et al., Astropart. Phys. 11 (1999) 287.

[16] D.J. Ficenec et al., Phys. Rev. D 36 (1987) 311.

[17] Evaluated Nuclear Data File (ENDF), National Nuclear Data Center, Brookhaven National Laboratory, 2001, http://www.nndc.bnl.gov.

[18] U. Kilgus, R. Kotthaus, E. Lange, Nucl. Instrum. Methods A 297 (1990) 425;

R. Kotthaus, Nucl. Instrum. Methods A 329 (1993) 433. 